Research Article

\title{
High-Capacity Real-Time Face Retrieval Recognition Algorithm Based on Task Scheduling Model for the Treatment Area of Hospital
}

\author{
Yi Zhou $\mathbb{D}$ and Weili Xia \\ School of Management, Northwestern Polytechnical University, Xi'an 710072, China \\ Correspondence should be addressed to Yi Zhou; zhouyi1202@mail.nwpu.edu.cn
}

Received 29 October 2021; Revised 17 November 2021; Accepted 22 November 2021; Published 6 December 2021

Academic Editor: Miaochao Chen

Copyright (C) 2021 Yi Zhou and Weili Xia. This is an open access article distributed under the Creative Commons Attribution License, which permits unrestricted use, distribution, and reproduction in any medium, provided the original work is properly cited.

\begin{abstract}
This paper presents an in-depth study of face detection, face feature extraction, and face classification from three important components of a high-capacity face recognition system for the treatment area of hospital and a study of a high-capacity realtime face retrieval and recognition algorithm for the treatment area of hospital based on a task scheduling model. Considering the real-time nature of our system, our face feature extraction network is modeled by DeepID, and the network is slightly improved by introducing a central loss verification signal to train a DeepID-like network model using central loss and use it to extract face features. To further investigate and optimize the schedulability analysis problem of the directed graph real-time task model, this paper proposes a rigorous and approximate response time analysis method for the directed graph real-time task model with an arbitrary time frame. Based on the theoretical results of the greatly additive algebra, it is shown that the coherent qualifying function is linearly periodic, i.e., the function can be represented by a finite nonperiodic part and an infinitely repeated periodic part, thus calculating the coherent qualifying function independent of the magnitude of the interval time. The algorithm for high-capacity real-time face retrieval and recognition in the treatment area of hospital based on the task scheduling model is further investigated, and a face database is established by using the PCA dimensionality reduction technique. Based on the internal architecture of the processor, image preprocessing and IP core packaging are implemented, and the hardware engineering of the high-capacity real-time face recognition system for hospital visits is built using the IPbased design concept. The performance tests of the face detection model and feature extraction network show that the face detection model has a significant reduction in false-positive rate, better fitting of border regression, and improved time performance. The face feature extraction network has no overfitting, and the features are highly discriminative with small feature extraction time consumption. The high-capacity real-time face recognition system for the treatment area of hospital combined with the optimized directed graph task scheduling model can approach $25 \mathrm{fps}$, which meets the real-time requirements, and the face recognition rate surpasses that of real people. It realizes the intelligence, self-help, and autonomy of medical services and satisfies the medical needs of users in all aspects.
\end{abstract}

\section{Introduction}

In the past half-century, biometric identification technology has developed rapidly because each biological individual has unique biometric points that can be measured, identified, and verified, and biometric identification technology identifies and authenticates individuals based on these unique points. Unlike traditional identification which is easily damaged, easily lost, and easily falsified, biometric identification technology which utilizes the unique characteristics of biological individuals is more convenient, fast, safe, reliable, and accurate. Biometric identification technology can be divided into two categories, one is identification by inherent characteristics of biological individuals, and the other is identification by behavioral characteristics of biologicals $[1$, 2]. Inherent characteristic identification currently has features like fingerprint, hand type, iris, retina, pulse, and face and has been used as biometric identification, and 
behavioral characteristics like voice and signature have been used as biometric identification. As a kind of biometric technology, face recognition has its unique advantages such as nonintrusiveness, convenience, friendliness, noncontact, and scalability compared with other biometric technologies in practical use scenarios, which makes it have an important position in biometric identification $[3,4]$. If the time constraints of the real-time system are met, the system is said to be schedulable, that is, each task is completed before its own time limit. Therefore, it is required to be able to determine in advance whether it is schedulable [5]. If the result is not schedulable, then you need to increase system resources or reduce task load so that the system becomes schedulable again.

Face recognition has become the current research hotspot in the field of artificial intelligence due to its huge application prospect. With the development of science and technology and the progress of society, the technical demand for conducting fast, efficient, and automatic face recognition is becoming more and more urgent. Face recognition technology first determines whether there is a face in the image or not, and if there is a face, the features of the face are extracted and matched with the known face comparison, and then, the identity of the face is recognized [6]. The face recognition process is generally divided into four steps: face detection, face feature extraction, face matching, and recognition. Among them, face feature extraction is the most important part of face recognition, and it is good or bad directly affects the final result of our face recognition. From another perspective, face recognition is finding a way to describe faces, and this description needs to have strong interclass distinguishability and intraclass stability. There are neural network-based methods, statistical methods, geometric feature-based methods, model-based methods, and multiclassifier integration methods for face recognition, among which the vast majority of traditional face recognition feature expressions are human selected; however, in practice, human selection of features is a very timeconsuming and energy-consuming matter, and the good or bad selection depends largely on experience and luck. However, with the increasing electronic, automated, and connected control systems, the functions and performance requirements undertaken by real-time systems are becoming more and more demanding. At the same time, the type, number, and a load of tasks in the system have increased dramatically, which poses a great challenge to the time verification of real-time systems [7]. Therefore, it is of great significance to research schedulability analysis and performance optimization of complex real-time task systems.

This paper will focus on the direction of the task scheduling model for a high-capacity real-time face retrieval recognition algorithm for hospital visits. The existing algorithms for large-capacity real-time face retrieval and recognition inhospital visit sites match sparse datasets, which makes the large-capacity heterogeneous face recognition inhospital visit sites cannot achieve the performance of visible face recognition when using the same task scheduling model. At the same time, the existing face recognition task scheduling model is large and cannot meet the needs of offline inference computation of edge devices nowadays. By investigating the multitask scheduling method to make the large-capacity real-time face recognition model complete the task quickly and accurately on the embedded platform is the purpose of this paper. Chapter 1 is the introduction, which briefly introduces the background and significance of the study of the real-time face recognition system and finally summarizes the main work of this paper and the structure of the paper. The second chapter is related work, briefly describes the status of domestic and international research, and both face detection and feature extraction are obtained using task scheduling model, and finally, it also introduces how to improve task scheduling performance by adjusting hyperparameters. Chapter 3 firstly introduces the research on the recognition algorithm of large-capacity real-time face retrieval inhospital visit sites based on task scheduling model; then, we select the current multitask scheduling model with very high real time and performance, improve it, and introduce better recognition methods for large-capacity real-time face retrieval inhospital visit sites. Chapter 4 is the result analysis, through the hospital visit place high-capacity real-time face retrieval recognition algorithm scheduling analysis, hospital visit place high-capacity real-time face retrieval recognition algorithm performance analysis, and hospital visit place high-capacity real-time face retrieval recognition algorithm system analysis, which proves the better performance and efficiency of the algorithm researched in this paper, the relevant test of our face recognition system, and the analysis of our face recognition system performance and result presentation. Chapter 5 concludes with a summary of the research work in this paper, pointing out the shortcomings of the scheme, proposing corresponding improvement methods, and looking ahead.

\section{Related Work}

Since individual task scheduling is computationally limited, most problems require multiple task schedulers to work together to solve the target problem, hence the emergence of multitasking scheduling systems. Multitask scheduling systems have considerable robustness and adaptability and are more efficient for solving practical application problems. Addagarla et al. combined binary particle swarm optimization with contract networks and used the synergy of task scheduling to improve the accuracy of high-capacity realtime face retrieval recognition and reduce the scheduling time in the treatment area of hospital. The dynamic path planning method is accomplished by using the dynamic and reactive nature of task scheduling and adding it to the ant colony algorithm [8]. Bartolini and Patella incorporated a microgrid composed of distributed power sources into a communication network formed by multitask scheduling technology, which enabled the system to ensure stable system power output even when the load changed drastically [9]. The multitask scheduling technique is used to coordinate and control the large-capacity real-time face retrieval and recognition in a hospital visit site, and the multitask scheduling technique is incorporated into it to enhance the communication function of the large-capacity real-time face retrieval and recognition in a hospital visit site and to 
maintain a trouble-free retrieval and recognition [10]. The information captured by task scheduling is processed by applying convolutional fusion. The task scheduling technique is incorporated into semantic Web services to make the discovery, invocation, and combination of Web services more efficient, and the multitask scheduling technique is introduced into deep reinforcement learning to solve the problem that the policy gradient increases with the number of task scheduling [11].

However, the rapid growth of face recognition technology in China in recent years and the booming of task scheduling have forced major research institutes, companies, and universities to invest heavily in face recognition [12]. Choi and Cha proposed a transformation algorithm using Eigen to reach the transformation from face sketch to face photo and then perform face matching on the face photo obtained from the change [13]. A method using locally linear embedding is proposed to transform the face sketch into a real photo image and the near-infrared light face image into a visible light face image, respectively, and then use a general method to match the face recognition [14]. To consider the relationship between face facial image regions and their neighboring regions in a comprehensive way, Markov random field model is added to the project of heterogeneous face recognition [15]. In this approach, since only the best candidate regions are selected for training, this leads to the deformation of facial images. Kumar et al. proposed a Markov weight field model, which can solve the problem of image deformation by selecting some selected regions to form a Markov network model [16]. Muhammad et al. proposed a direct push learning method using Markov random fields, which combines sketch images with real photo images of the test images into the learning process, thus reducing the error of the model on the test data [17].

This paper proposes and studies a task model for dynamically adjusting the switching speed, which is a strict extension of the static adaptive rate-of-change task model [18]. A schedulability analysis method was developed for such a new real-time task model. This method introduces a new directed graph task model to safely approximate the execution load of this type of rotation task. Compared with the existing static analysis methods, the proposed schedulability analysis method has higher accuracy and can effectively reduce the time complexity [19]. Hospital visit site high-capacity real-time face retrieval recognition is a process algorithm used to describe concurrent systems, and its simple and efficient algorithmic model makes hospital visit site high-capacity real-time face retrieval recognition the basis for modeling in several fields. The CCS is promoted for high-capacity real-time face retrieval recognition at hospital sites and can be used to represent communication topologies with dynamic change capability by transmitting channels between distributed nodes along other channels, which is very powerful for formal description. Since a change in the physical location of processors in a distributed system may bring about a change in the communication topology, it is particularly suitable to describe a concurrent programming model for task scheduling using high-capacity real-time face retrieval recognition inhospital visit sites. The goal of this paper is to combine static task scheduling algorithms with multitask scheduling techniques and high-capacity realtime face retrieval recognition inhospital visits, so that task scheduling in a distributed environment can better utilize environmental resources, not only to enhance concurrency and reduce communication overhead by using multitask scheduling techniques but also to combine the simulated communication capability of high-capacity real-time face retrieval recognition inhospital visits [20]. It defines a task scheduling model for uncertainty-oriented concurrent systems that reacts faster to network topology changes and is important for efficient utilization of resources in distributed systems.

\section{Intelligent Analysis of Supply Chain Coordination Based on the Internet of Things}

3.1. High-Capacity Real-Time Face Information Detection and Extraction for Hospital Consultation Places. In the face of multipose face detection task, the organization of the classifier is an important issue, especially when the features of faces in different poses show great differences, which will bring a great challenge to the detector; to solve this problem, a partitioning strategy is usually used, and the classifiers are trained separately for faces in different poses and then combined to build a multipose face detector. The partitioning strategy is the most basic strategy to deal with the multipose face detection task, but it also needs to take into account the speed and classification accuracy, and the enhancement of classification capability will bring an increase in computational cost.

We design Equation (1) by combining the localization error Dc and classification error Fc of the samples, $y$ the prediction probability of classification, and $x$ the intersection rate between the border and the actual target border and calculate a surrogate value cost for the border containing positive samples in the grid, based on which we judge whether the sample is selected to join the training.

$$
C(x, y)=\operatorname{Dc}(x)+\operatorname{Fc}(y) .
$$

The positioning error Dc is defined as Equation (2).

$$
\operatorname{Dc}(x)=\frac{1}{1+\lim _{S \longrightarrow \infty} \sum_{i=1}^{S} i(x-t)(x+t)} .
$$

The classification error Fc is defined as Equation (3).

$$
\operatorname{Fc}(y)=(1-y) \lim _{S \longrightarrow \infty} \sum_{i=1}^{S} i(y-t)(y+t) .
$$

In Equations (2) and (3), $t$ is a certain threshold value of the intersection rate. $i$ is a coefficient that controls the sensitivity of the localization error Dc to the threshold value $t$; the inputs $x$ and $y$ for both costs take values between 0 and 1 . 


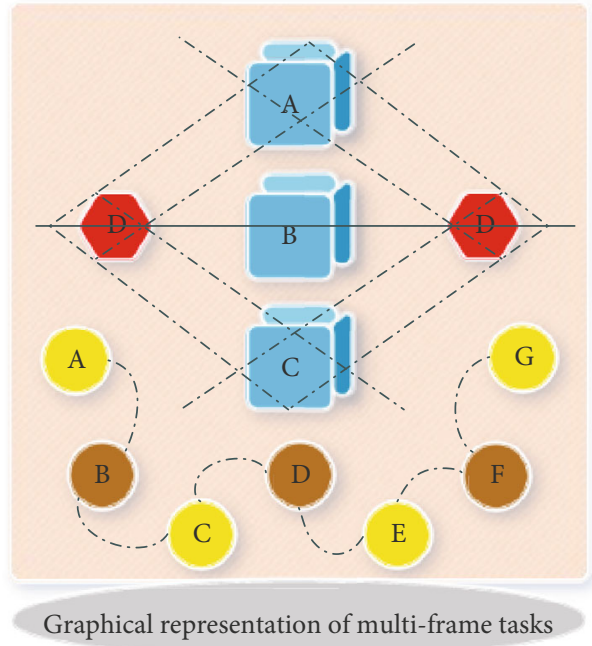

(a)

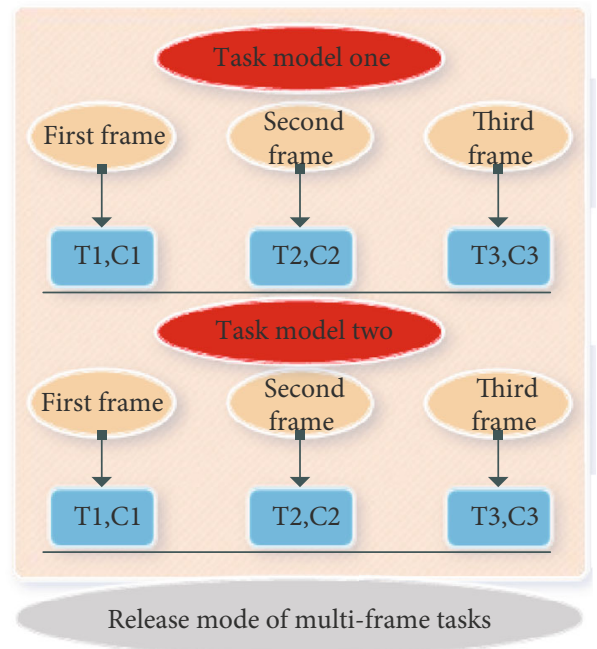

(b)

FIGURE 1: Graph representation and release pattern for multiframe tasks.

The purpose of DeepID2 for learning is to obtain the output vector, not to maximize the recognition rate; thus, the class spacing term is added to the paper. To facilitate the representation, we express the output vector obtained by DeepID2 layer extraction as a function, as in Equation (4), where $T(x)$ denotes the extracted DeepID2 feature vector, the $t$ function denotes the feature extraction function, $x$ denotes the input face slice, and $\beta$ denotes the network parameters.

$$
T(x)=t(x, \beta)
$$

The function for correct classification is the objective function of softmax, which is aimed at minimizing the cross-entropy and is defined to identify the signal, as in Equation (5), where $T$ is the DeepID2 feature vector of Equation (4), $t$ denotes the classification target, $\beta_{i}$ denotes the softmax layer parameter, $p(i)$ denotes the target probability distribution, and $p(t)$ denotes the classification prediction probability distribution.

$$
S\left(T, t, \beta_{i}\right)=\lim _{N \longrightarrow \infty} \sum_{i=1}^{N} p(i) \ln p(i)=-\ln p(t) .
$$

The objective function for maximizing the sample spacing is as follows, defined as the validation signal, as in Equation (6), where $s_{i j}=1$ means that $f i$ and $f j$ belong to the same person, and this is when we need to minimize the interclass spacing; $s_{i j}=-1$ means that $T_{i}$ and $T_{j}$ belong to different people, and this is when we need to minimize the difference between $m$ and their distance values. The $m$ is a parameter that needs to be adjusted manually, and the purpose of proposing $m$ is that the objective function needs to be minimized, not maximized. Since the validation signal needs two samples to be calculated, the training process of the whole network needs to be changed accordingly. Two samples are randomly selected for each iteration during training and then trained. Our goal is to learn the parameter $\beta_{i j}$ updated by stochastic gradient descent.

$$
H\left(T_{i}, T_{j}, s_{i j}, \beta_{i j}\right)=\frac{\max \left(0,\left|T_{i}-T_{j}\right|^{2} / 2, m-\left|T_{i}-T_{j}\right|^{2}\right)}{2} .
$$

\subsection{High-Capacity Real-Time Face Retrieval Recognition} Algorithm Based on Task Scheduling Model for the Treatment Area of Hospital. The multiframe task $i$ can be described by the minimum release interval $T i$, the execution time vector $C i=(C i, 1 ; \cdots \cdots ; C i, m i)$, and the relative time frame $D i=T i$. A typical example of a multiframe task model is an MPEG video stream decoding application using multiple frame types, capable of simulating the periodic arrival of video frames and different frames with different decoding times. As shown in Figure 1, a multiframe task can be represented by a graph with a chain structure (Figure $1(\mathrm{a})$ ), where the solid nodes represent the entry points for task execution. In addition, the execution time of the multiframe task is variable, but the release pattern and the relative time frame are fixed (Figure 1(b)).

For $n$ independent multiframe tasks running on a processor with a scheduling policy using the preemptive static priority RM algorithm, the system is schedulable if Equation (7) is satisfied.

$$
U(x)=\left[\left(1+\frac{1}{x}\right)^{1 / n}-1\right](x+n)(x-n) .
$$

Each node $x_{i} \in X$ (or task type) is represented by an ordered pair $\left\langle e\left(x_{i}\right), d\left(x_{i}\right)\right\rangle$, where $e\left(x_{i}\right)$ and $d\left(x_{i}\right)$ are the worst-case execution time and the relative time frame, respectively. The edges represent the possible control flow directions, i.e., the order of releasing tasks. An edge $\left(x_{i}, y_{j}\right)$ $\subseteq K$ is described by the parameter $p\left(x_{i}, y_{j}\right)$, which is the 
minimum release interval time for $x_{i}$ and $y_{j}$. We assume that all parameters are positive integers, and that the WCET of each node is not greater than the minimum release interval of all its outgoing edges, i.e., it satisfies Equation (8).

$$
\exists\left(x_{i}, y_{j}\right) \subseteq K, e\left(x_{i}\right) \doteq p\left(x_{i}, y_{i}\right)
$$

For the same path $t=\left\{x_{1}, x_{2} \cdots \cdots x_{n}\right\}$ in task $T$ functions $f$, there exists a vertical to slope one substitution relation defined as follows: for any vertical part of $f$ as in Equation (9).

$$
f(T, x)=T * f(t)+\psi
$$

For task $T$, the maximum cumulative execution request of its task release in an interval of arbitrary length $t$ is defined as its request-limited function, as in Equation (10).

$$
g(T, x)=\max _{\beta \subseteq D(T)}(T f(t)(1-\beta)(1+\beta)) .
$$

For task $T$, its task release is defined as its coherencelimited function in terms of the maximum number of execution loads in an interval of arbitrary length $t$, as in Equation (11).

$$
h(T, x)=\max _{\beta \subseteq D(T)}\left(\operatorname{Tg}(t)\left(\frac{(1-\beta)(1+\beta)}{\lim _{N \longrightarrow \infty} \sum_{i=1}^{N} \beta_{i}}\right)\right) .
$$

For task $T$, whose tasks are released and the time frame is within interval $t$, its maximum cumulative execution demand is defined as its demand-limited function, as in Equation (12).

$$
y(T, x)=\max _{\beta \subseteq D(T)}(h(t)(1-\beta)(1+\beta)) .
$$

Intuitively, the $f()$ and $g()$ functions quantify the maximum cumulative execution request and load, respectively, while the $y()$ function measures the size of the execution time that a task is released and must be completed within a given interval. Like the $f()$ function, the $g()$ function is a nondecreasing step function whose horizontal part is leftopen and right-closed. At the same time, the $y()$ function inherits the properties of $y$ and is also a continuous sloping step function. Like the $g()$ function, the $g()$ function is a step function whose horizontal part is left-closed and rightopen.

To reduce the complexity of computation and to achieve the purpose of real-time processing, dimensionality reduction methods are used in face recognition. The commonly used mainstream linear transformation method for dimensionality reduction is principal component analysis (PCA), which is derived from $\mathrm{K}-\mathrm{L}$ transformation, and its essence is to protect the data in the high-dimensional space into the low-dimensional space through a linear transformation while representing the original data as well as possible. In the process of real-time face recognition, assuming that the fps (frames per second) of the currently captured video is $30 \mathrm{~Hz}$ and the resolution of the video image is $m$. Then, at a certain time, there will be $n$ images, and $m$ is the dimension of each image. That is, in the $m$-dimensional space, there exist $n$ samples as in Equation (13). The dimensionality of the data is too high, it is time-consuming and laborious to process, so I wonder if I can only process part of the dimensions, and the results obtained are consistent with the results of all dimensions. To put it simply, a picture has 2000 feature dimensions, but in fact, only 100 of them (or even less) have a huge impact on the result.

$$
M=\lim _{n \longrightarrow \infty}\left|\sum_{i=1}^{n} x_{i}\right|_{n}
$$

Assuming that 1 line is to be used to represent these $n$ samples and let $e$ denote the unit direction vector of this line through the mean, the equation of the line can be expressed as Equation (14), where $a$ is a real scalar representing the distance of a point on the line from the point $m$.

$$
d(x)=d(m)+\alpha * \psi
$$

A linear projection of the $n$ samples in the direction of the eigenvector corresponding to the maximum eigenvalue of the walk matrix yields a 1-dimensional representation $i$ a in the least square error sense, and this projection transformation is the transformation of the bases. In the $m$-dimensional space, the $m$ bases are unit vectors $\psi_{i}$ in the direction of each coordinate axis $(i=1,2 \cdots n)$, and a certain sample $x_{i}\left(x_{i 1}, x_{i 2} \cdots x_{i m}\right)$ in the space can be represented by this set of bases as Equation (15).

$$
x_{i}=\lim _{m \longrightarrow \infty} \sum_{i=1}^{m} x_{i j} \psi_{i}+\lim _{n \longrightarrow \infty} \sum_{j=1}^{n} x_{i j} \psi_{i} .
$$

A nonlinear activation function with a segmented linear activation function (Hard_Swish) is introduced in the Lightfacenet model, which can significantly improve the accuracy of the neural network for classification tasks when using Hard_Swish instead of as ReLU, which is improved from the Swish nonlinear activation function. Swish is defined in Equation (16).

$$
\text { Swish }=\lim _{M \longrightarrow \infty} \sum_{i=1}^{M=m n}\left(x_{i}-x_{i-1}\right)^{2} .
$$

The bottleneck is a bottleneck structure, which can be used in the network to reduce the number of parameters and computation of the network with no loss of accuracy. The structure is shown in Table 1.

In the traditional method, the Euclidean distance is usually used to determine the identity similarity has a great limitation. The characters classified by the softmax function will have two different identities that are closer than the same identity, thus failing to achieve the classification effect, and adding intraclass constraints through the cross-entropy loss 
TABLE 1: Bottleneck structure.

\begin{tabular}{lccc}
\hline Serial number & Input & Operate & Output \\
1 & $h \times w \times d$ & Conv $1 \times 1$ & $h \times w \times d$ \\
2 & $h \times w \times$ Ltd & Conv $2 \times 2$ & th $\times w \times$ Ltd \\
3 & $h \times \mathrm{tw} \times d$ & Conv $3 \times 3$ & th $\times \mathrm{tw} \times d$ \\
4 & th $\times w \times d$ & Conv $4 \times 4$ & th $\times \mathrm{tw} \times \mathrm{Ltd}$ \\
\hline
\end{tabular}

function can make the network have a better classification effect, and the improved formula is shown in Equation (17).

$$
Y=\ln \frac{\psi^{W(x)}(\beta+1)(\beta-1)}{\beta \lim _{n \longrightarrow \infty} \sum_{i=1}^{n} \psi^{W(x)+x_{i}}} .
$$

By normalizing the weights and features, the prediction of the network depends only on the learning of the weights and feature angles $\psi$. All features are distributed on a hypersphere of radius $s$. A hyperparameter is added to the crossentropy loss function for the edge penalty of the weights and feature angles $\psi$. This is done to increase the edge angles between categories and at the same time make the categories more compact internally, resulting in a better performance for facial recognition, as in Equation (18).

$$
Y=\ln \frac{\psi_{n \longrightarrow \infty}^{\sin \left(x_{i}+T\right)}}{\lim _{n \longrightarrow} \sum_{i=1}^{n} \psi^{W(x)+x_{i}}+\lim _{n \longrightarrow \infty} \sum_{i=1}^{n} \psi^{\sin \left(x_{i}+T\right)}} .
$$

If two task instance sequences have the same task instance sequence, then as far as the coherence function is concerned, one task instance sequence with the shortest release interval will dominate the other task instance sequences. Therefore, we can divide the release time area of the task instance so that each node is fixed in each release interval.

3.3. Design of High-Capacity Real-Time Face Retrieval System for Hospital Consultation Places. The design of the hospital visit place high-capacity real-time face retrieval system architecture is oriented to all users, the overall goal of the system as the basis of the design, standing in the global overview perspective of the system's overall perspective for the construction of the hospital visit place high-capacity real-time face retrieval of the business aspects of the needs and technical aspects of the needs from multiple management and constraints, from a comprehensive perspective requires the construction of a unified platform, the global model with unified specifications. The system is designed for a five-layer structure, as shown in Figure 2.

The planning analysis is mainly carried out in two aspects: on the one hand, it is data-oriented and analyzes the current situation of data application for each department and each data source; the data quality analysis is mainly focused on the usability of data and the usage of data. Based on data application status and data quality analysis, the system's shared data model is constructed by combining application analysis. From another aspect, planning analysis is oriented to applications and processes, and planning analy- sis is conducted for applications, processes, and data exchange and sharing involving cross-department and cross-system, and on top of that, an application process model is constructed. Based on these two aspects, a feasible planning and analysis report is formed and the outline design and detailed solution design are written and loaded into the integration and development and implementation phase of the project.

The self-built face database contains the face data of 5 people in the lab, in which each person's face data contains 10 images with different angles and each face image has a pixel size of $112 * 92$. The self-built face database uses 9 face images of each person as training and 1 face image as testing. In this paper, the validation of the PCA algorithm dimensionality reduction is implemented by Matlab. Firstly, the data from the self-built face database is read, and the sample matrix is generated; secondly, the dimensionality reduction is implemented by the PCA algorithm, and finally, the principal component faces are displayed. The main parameters include the number of people read in, the number of samples nFacePerPerson read in for each person, and the container FaceContainer to store the sample matrix. In the process of implementing the PCA algorithm, the difficulty is to calculate the sample covariance matrix of eigenvalues and eigenvectors. The custom function named CalculationPCA calculates the eigenvectors and eigenvalues of the matrix by calling the library function. The input parameters are the stored sample matrix FaceContainer and the dimension $K$ to be reduced to; the output parameters are the sample eigenvector matrix and the principal component vector after reducing to $K$ dimensions; finally, the reduced dimensional principal component face is displayed. The output of the reduced dimensional data is saved. Since this paper adopts the functions of OpenCV to realize the detection and recognition function of the human face, the XML file is completed by OpenCV to ensure uniformity.

When a noisy image is acquired, the next step is to process the image to achieve the desired effect. Second, to increase the detectability of the object to be detected in the image and to improve the image quality, grayscale processing, filtering, noise reduction, and contrast enhancement operations are required for the acquired image. The ARM architecture has increased the clock speed by simplifying the instruction set and also uses pipelining to increase the speed of the processor instruction stream, but the nature of ARM for image processing is serial, especially for embedded low-power products, due to power constraints, the clock speed needs to be reduced, which makes it difficult to ensure the real-time requirements. This is where the significant acceleration of many image processing algorithms can be achieved. In this paper, we take advantage of the high-speed parallelism of FPGAs to perform grayscale processing, median filtering, and histogram equalization on the acquired images through the PL part of Zynq and package the module as an IP core.

\section{Results and Analysis}

4.1. Scheduling Analysis of High-Capacity Real-Time Face Retrieval Recognition Algorithm for Hospital Consultation 


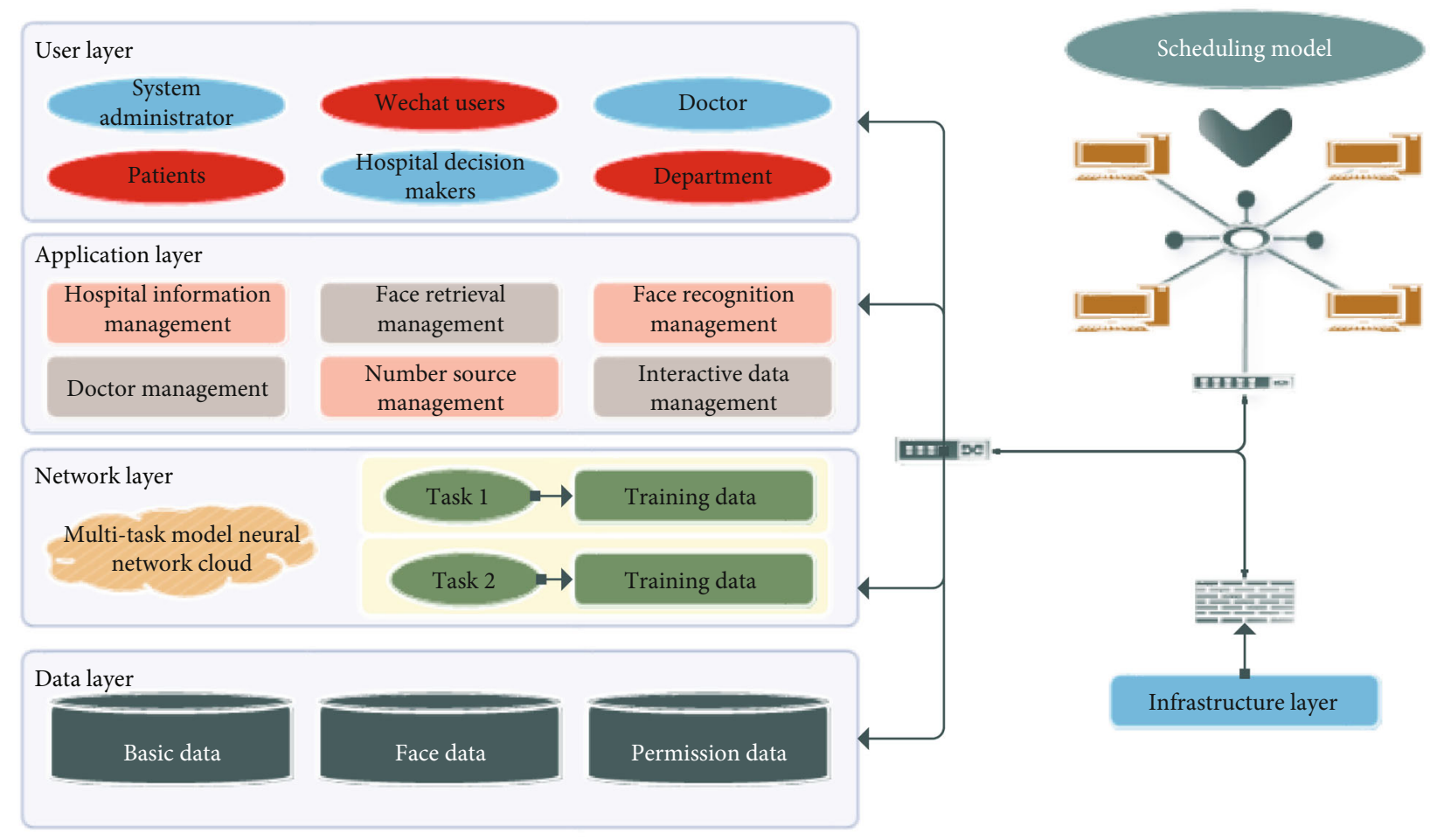

Figure 2: Overall system architecture diagram.
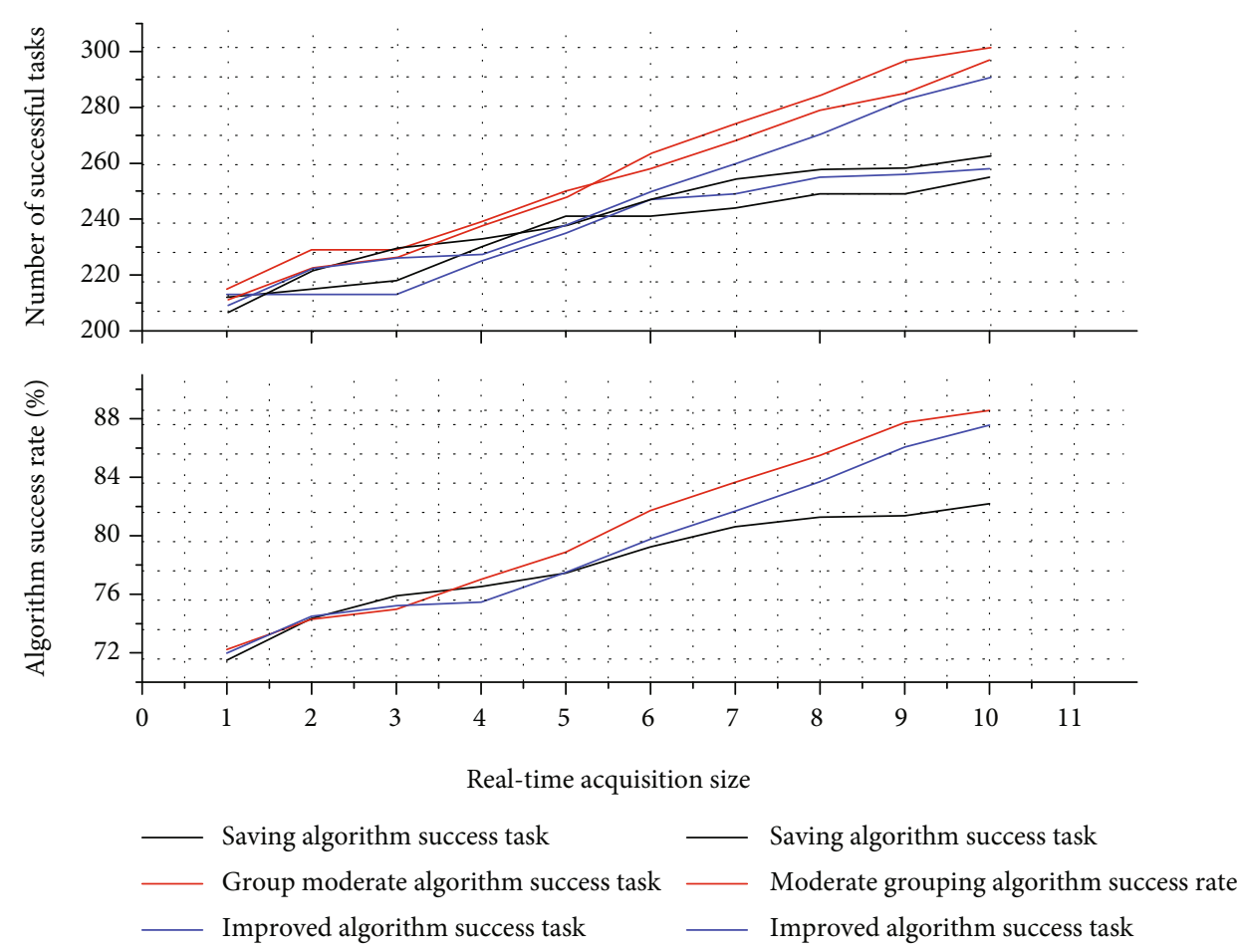

FIGURE 3: Comparison of scheduling success rate of different scheduling algorithms.

Places. As shown in Figure 3, the scheduling success rate of both the grouped moderate algorithm and the improved algorithm is higher than the saving algorithm in the same feasibility check window. When the Wnd value is small, the scheduling success rate of the improved algorithm is not as good as that of the grouped moderate algorithm, but as the Wnd value increases, the success rate of the improved algorithm increases and exceeds that of the grouped 


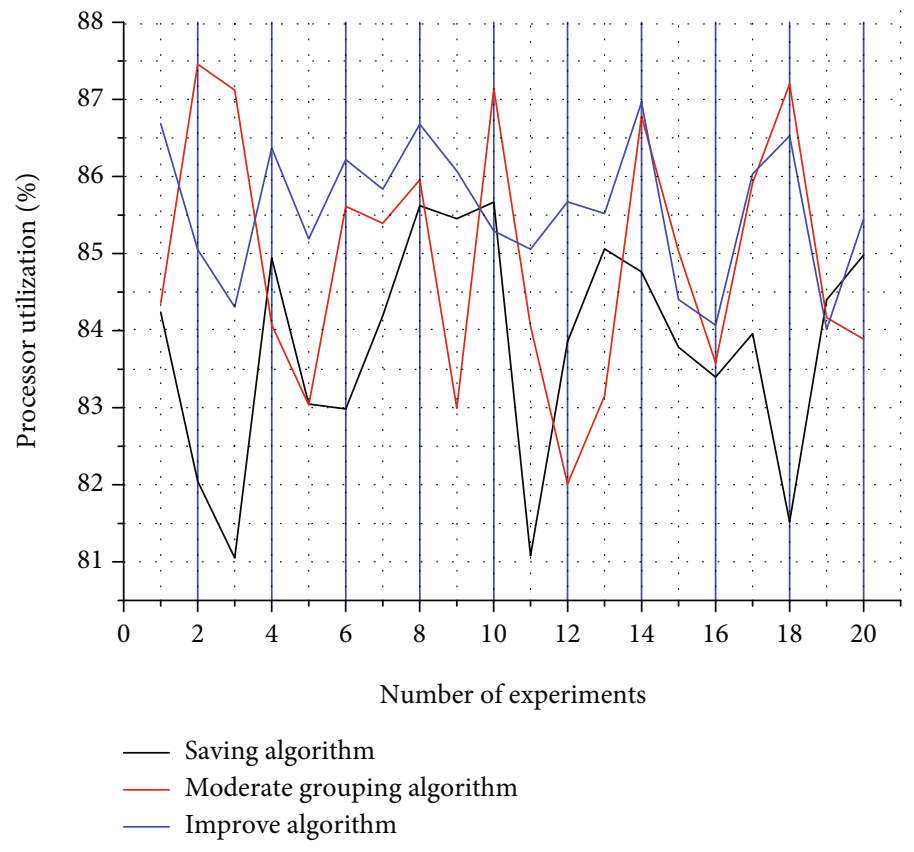

Figure 4: Processor utilization of the algorithm.

moderate algorithm when Wnd is equal to 5 . This is because the improved algorithm needs to group the tasks in the window, and the grouping algorithm of the improved algorithm is more sensitive to the size of the feasibility check window because it takes into account the sharing of all resources of the tasks compared to the grouping method in the grouping moderate. When the window value is small, the difference between the improvement algorithm and the saving algorithm is not too big, and the effect of grouping will slowly appear when the window value becomes larger.

It can be seen from Figure 4 that among the processor utilization rates of several algorithms, the average core utilization rate of the improved algorithm is higher than that of the other two, but the improved algorithm fluctuates more sharply than other algorithms. After calculation, the average utilization rate of the saving algorithm, the appropriate grouping algorithm, and the improved algorithm is $82.32 \%, 82.76 \%$, and $86.12 \%$, and the standard deviations are $2.18,1.64$, and 3.25 , respectively. One reason for this situation is that the improved algorithm will try to put tasks with a high degree of correlation into the same core as much as possible, but this will lead to a decrease in the degree of load balancing.

4.2. Performance Analysis of High-Capacity Real-Time Face Retrieval Recognition Algorithm for Hospital Consultation Places. To validate the task scheduling-based high-capacity real-time face retrieval and recognition algorithm for hospital visits, the neural network framework is used to implement the algorithm result analysis, and comparative experiments are done in the training phase of the network in terms of network structure, the number of parameters, running speed, and recognition rate. The training samples of the data set were divided by $11: 2$ to generate the training

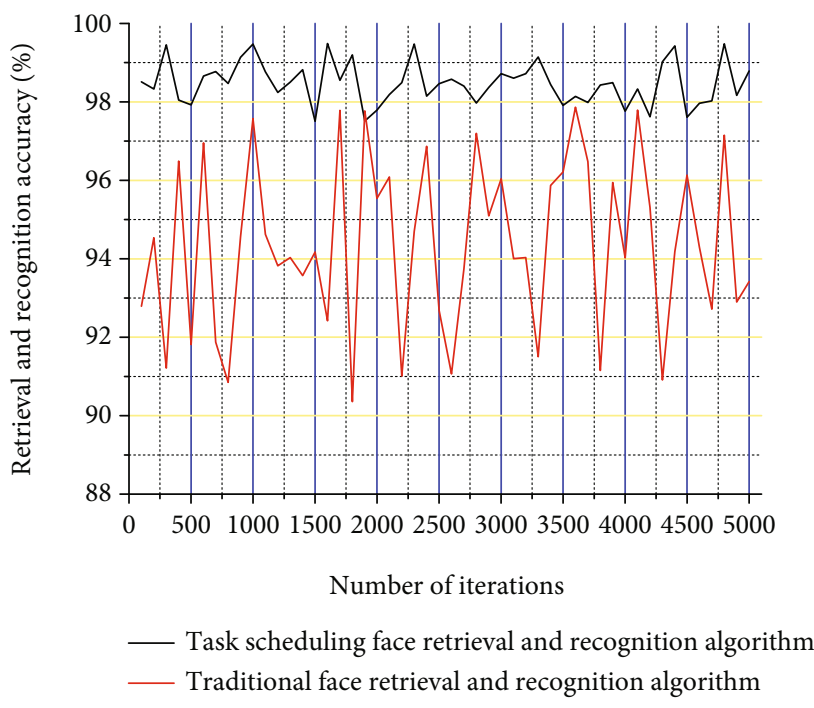

FIgURE 5: Algorithm retrieval recognition effect.

set and test set for the experiments. The comparison results of the experimental results are shown in Figure 5.

The time required by the task scheduling model-based high-capacity real-time face retrieval recognition algorithm and the traditional real-time face retrieval recognition algorithm in the hospital visit site were tested separately. As shown in Figure 6, it can be seen that the time required by the traditional real-time face retrieval recognition algorithm is about three times longer than that required by the task scheduling model-based high-capacity real-time face retrieval recognition algorithm for hospital visits, and it can be seen that the speed of face detection is improved by the task scheduling model, and the design objective is achieved. 


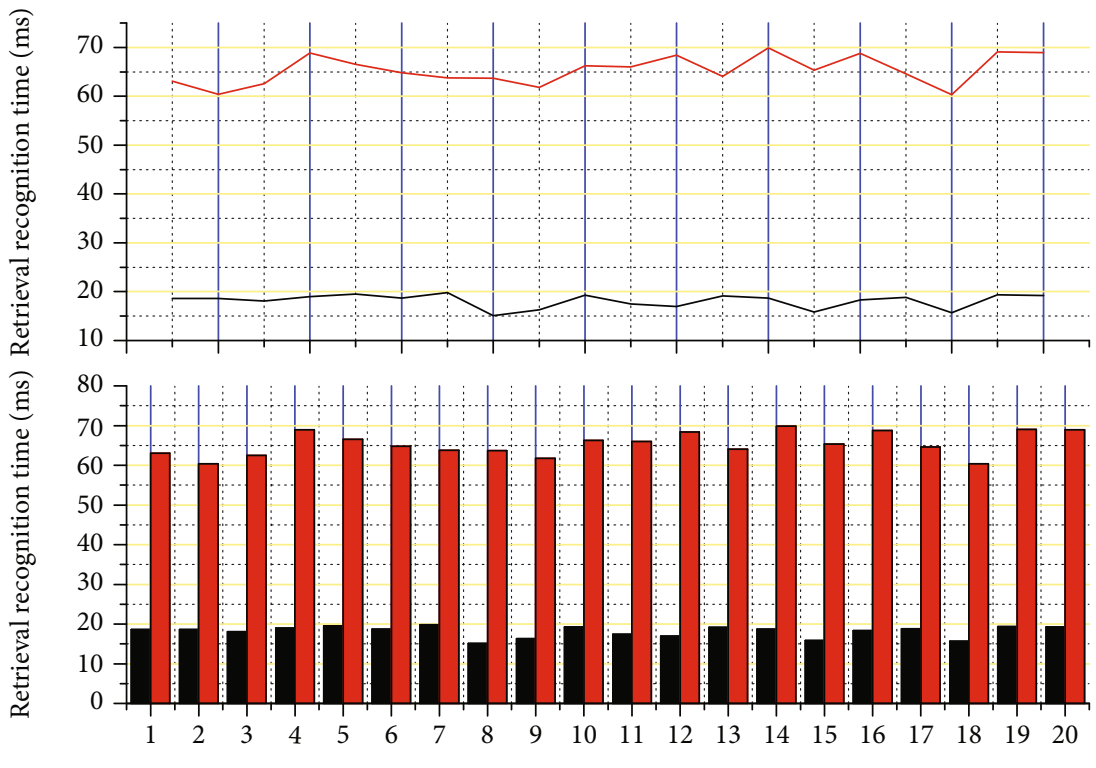

Retrieval recognition times

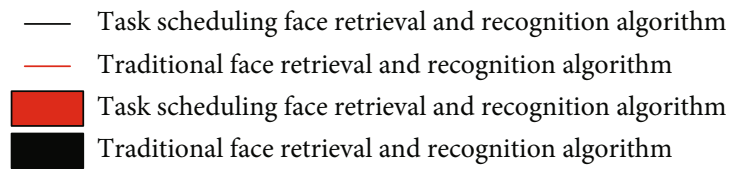

Figure 6: Time required for face detection.

\subsection{Analysis of High-Capacity Real-Time Face Retrieval} Recognition Algorithm System for Hospital Consultation Places. The features extracted by the feature extraction network are subjected to SVM classification to see the overall recognition performance of the network, and we perform two experiments to verify the network performance. We first extract the database of 1000 people and select the database containing 5 to 100 a priori face images to extract face features to train the SVM classifier, leave 5 images for each person as a test to test the classification performance, and test the average value three times, respectively, and the results are shown in Figure 7. As can be seen in Figure 7, our network recognition performance increases in classification accuracy in the early stage according to the increase of a priori pictures, the highest reached $98.76 \%$.

In the test set, we use CASIA Face dataset for testing, and the network is based on task scheduling to do migration learning. This experiment will be done by covering more combinations randomly and uniformly before the training starts, and the anchor points can be NIR or visible images. The positive samples have the same id as the anchor points, and the subsamples have different ids from the anchor points, according to which about 160,000 sets of training data can be obtained. Using these data, the trained visible face recognition network is set up as a three-channel network structure, and the network is trained by a triple loss function, and the batch size is set to 256 and goes in for training. The experimental results are shown in Figure 8. From Figure 8, it can be seen that the scheme in this paper has surpassed the existing mainstream algorithmic schemes. When using multitask scheduling as the base network for

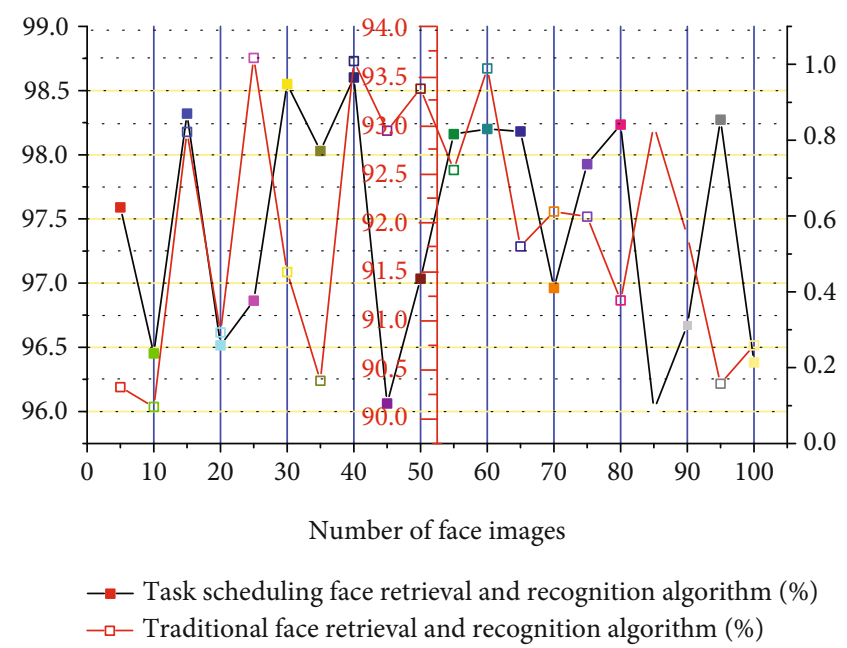

Figure 7: System recognition performance.

training, the recognition rate in the CASIA Face dataset reaches a level of $99.2 \%$ and the verification rate reaches $98.9 \%$. When using the traditional method as the base network, the recognition rate reaches $98.8 \%$ and the verification rate reaches $98.5 \%$ in the dataset. The task scheduling network structure in this paper achieves similar performance while using a fewer number of parameters and computations than a standard convolutional network such as the traditional network. This results in a high-capacity real-time face recognition network for hospital visits, which illustrates the method of pretraining a perfect face recognition model by task scheduling the face dataset and then using a triple loss 


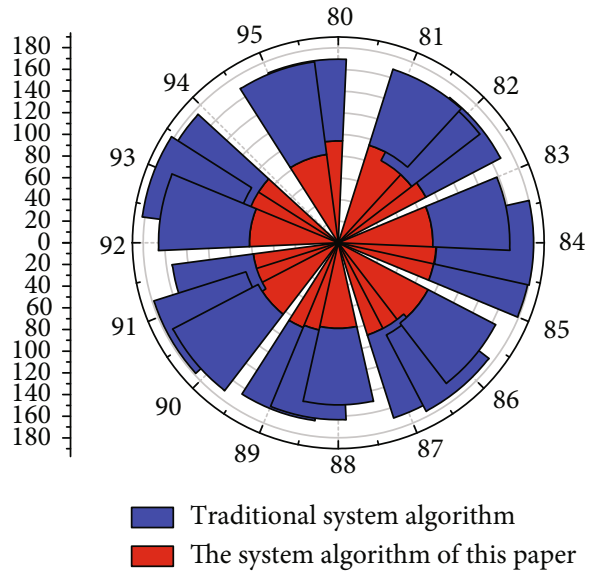

Figure 8: Face recognition experiment results.

function with a suitable triplet for small-scale datasets for migration learning, which has a good effect for learning small-scale datasets.

\section{Conclusion}

This paper investigates the task scheduling-based highcapacity real-time face retrieval recognition algorithm for hospital visit places, which is not convenient for the equipment due to a large number of scheduling parameters. The algorithm of large-capacity real-time face retrieval and recognition for hospital consultation places based on task scheduling is proposed. Firstly, the weaknesses and shortcomings of the existing commonly used algorithms in doing face recognition are analyzed, and they are improved in terms of the multitask scheduling network structure. A multitask scheduling approach is proposed to replace the existing high-capacity real-time face retrieval inhospital consultation places to recognize facial information with different degrees of importance in different locations. For the triple loss function training method, the impact of poor training data selection and construction method on neural network training is analyzed, and a reasonable method of training data triad selection and construction is proposed. A reasonable training data triad selection and construction method are proposed to solve the problem that the network cannot converge quickly due to the random selection of data to build the training set and then do migration learning with the triple angular loss function to improve the training effect of the network. Further, the acceleration factors between RPM divisions with different granularity are investigated and abounded upper bound on the acceleration factors of two effective RPM divisions with subset relations which are given to demonstrate the bounded pessimism of the proposed analysis method. The core of the algorithm is the use of hybrid schedulability analysis techniques with different accuracy and complexity. Experimental results show that the optimization method proposed in this chapter provides better and near-upper bound performance while significantly reducing the running time of the algorithm compared to the best available related research results. Although the system designed in this paper improves the speed of face detection and combines soft and hard codesign to accomplish the face recognition function, there are still some shortcomings. Firstly, it is difficult to improve the recognition rate of face recognition by relying on the simple task scheduling algorithm, and the SVM algorithm will be added in the next work to improve the recognition rate of face recognition. These shortcomings will be improved in future work and practice.

\section{Data Availability}

The data used to support the findings of this study are available from the corresponding author upon request.

\section{Conflicts of Interest}

The authors declare that they have no known competing financial interests or personal relationships that could have appeared to influence the work reported in this paper.

\section{References}

[1] P. Porambage, J. Okwuibe, M. Liyanage, M. Ylianttila, and T. Taleb, "Survey on multi-access edge computing for internet of things realization," IEEE Communications Surveys \& Tutorials, vol. 20, no. 4, pp. 2961-2991, 2018.

[2] Z. H. Ali and H. A. Ali, "Towards sustainable smart IoT applications architectural elements and design: opportunities, challenges, and open directions," The Journal of Supercomputing, vol. 77, no. 6, pp. 5668-5725, 2021.

[3] E. Indrawan, N. Jalinus, and S. Syahril, "Project based learning in vocational technology education study of literature," International Journal of Scientific \& Technology Research, vol. 9, no. 2, pp. 2821-2825, 2020.

[4] S. Abirami and P. Chitra, "Energy-efficient edge based realtime healthcare support system," Advances in Computers, vol. 117, no. 1, pp. 339-368, 2020.

[5] W. M. Alenazy and A. S. Alqahtani, "Gravitational search algorithm based optimized deep learning model with diverse set of features for facial expression recognition," Journal of Ambient Intelligence and Humanized Computing, vol. 12, no. 2, pp. 1631-1646, 2021.

[6] X. Zhang, W. Huang, X. Lin, L. Jiang, Y. Wu, and C. Wu, "Complex image recognition algorithm based on immune random forest model," Soft Computing, vol. 24, no. 16, pp. 12643 12657, 2020.

[7] S. Baek, "System integration for predictive process adjustment and cloud computing-based real-time condition monitoring of vibration sensor signals in automated storage and retrieval systems," The International Journal of Advanced Manufacturing Technology, vol. 113, no. 3-4, pp. 955-966, 2021.

[8] S. K. Addagarla, G. K. Chakravarthi, and P. Anitha, "Real time multi-scale facial mask detection and classification using deep transfer learning techniques," International Journal, vol. 9, no. 4, pp. 4402-4408, 2020.

[9] I. Bartolini and M. Patella, "A general framework for real-time analysis of massive multimedia streams," Multimedia Systems, vol. 24, no. 4, pp. 391-406, 2018.

[10] F. M. Talaat, S. H. Ali, A. I. Saleh, and H. A. Ali, "Effective load balancing strategy (ELBS) for real-time fog computing 
environment using fuzzy and probabilistic neural networks," Journal of Network and Systems Management, vol. 27, no. 4, pp. 883-929, 2019.

[11] Y. Xu, S. Wu, M. Wang, and Y. Zou, "Design and implementation of distributed RSA algorithm based on Hadoop," Journal of Ambient Intelligence and Humanized Computing, vol. 11, no. 3, pp. 1047-1053, 2020.

[12] Q. Zhang, H. Sun, X. Wu, and H. Zhong, "Edge video analytics for public safety: a review," Proceedings of the IEEE, vol. 107, no. 8, pp. 1675-1696, 2019.

[13] W. Choi and Y. J. Cha, "SDDNet: real-time crack segmentation," IEEE Transactions on Industrial Electronics, vol. 67, no. 9, pp. 8016-8025, 2019.

[14] L. J. Rubini and E. Perumal, "Hybrid kernel support vector machine classifier and grey wolf optimization algorithm based intelligent classification algorithm for chronic kidney disease," Journal of Medical Imaging and Health Informatics, vol. 10, no. 10, pp. 2297-2307, 2020.

[15] C. Deng, R. Guo, C. Liu, R. Y. Zhong, and X. Xu, "Data cleansing for energy-saving: a case of cyber-physical machine tools health monitoring system," International Journal of Production Research, vol. 56, no. 1-2, pp. 1000-1015, 2018.

[16] S. N. Kumar, A. L. Fred, and P. S. Varghese, "An overview of segmentation algorithms for the analysis of anomalies on medical images," Journal of Intelligent Systems, vol. 29, no. 1, pp. 612-625, 2018.

[17] G. Muhammad, M. F. Alhamid, and X. Long, "Computing and processing on the edge: smart pathology detection for connected healthcare," IEEE Network, vol. 33, no. 6, pp. 44-49, 2019.

[18] S. Chen, Z. Zhang, J. Yang et al., "Fangcang shelter hospitals: a novel concept for responding to public health emergencies," The Lancet, vol. 395, no. 10232, pp. 1305-1314, 2020.

[19] S. Jain, R. Khera, Z. Lin, J. S. Ross, and H. M. Krumholz, "Availability of telemedicine services across hospitals in the United States in 2018: a cross-sectional study," Annals of Internal Medicine, vol. 173, no. 6, pp. 503-505, 2020.

[20] P. Yang, K. M. Treurniet, L. Zhang et al., "Direct intra-arterial thrombectomy in order to revascularize AIS patients with large vessel occlusion efficiently in Chinese tertiary hospitals: a multicenter randomized clinical trial (DIRECT-MT) - protocol," International Journal of Stroke, vol. 15, no. 6, pp. 689-698, 2020. 